\title{
Heegaard and Regular Genus of 3-Manifolds with Boundary
}

\author{
P. CRISTOFORI, C. GAGLIARDI and L. GRASSELLI
}

\begin{abstract}
By means of branched coverings techniques, we prove that the Heegaard genus and the regular genus of an orientable 3-manifold with boundary coincide.
\end{abstract}

\section{PRELIMINARIES}

Throughout this paper the term "manifold" will denote a compact, connected, orientable PL-manifold with (possible empty) boundary.

An $(n+1)$-coloured graph (with boundary) is a pair $(\Gamma, \gamma)$, where $\Gamma=(V(\Gamma), E(\Gamma))$ is a multigraph and $\gamma: E(\Gamma) \rightarrow \Delta_{n}=\{0,1, \ldots, n\}$ is a map such that $\gamma(e) \neq \gamma(f)$, for each pair $e, f$ of adjacent edges of

(*) Work performed under the auspices of the G.N.S.A.G.A. of the C.N.R. (National Research Council of Italy) and within the project "Geometria Reale e Complessa", supported by M.U.R.S.T. of Italy

1991 Mathematice Subject Classification: 57Q15, 57M12, $05 \mathrm{C15}$.

Servicio publicaciones Univ. Complutense. Madrid, 1995. 
$\Gamma ; \gamma$ is called edge-coloration on $\Gamma$. For each $B \subseteq \Delta_{n}$, the $B$-residues of $(\Gamma, \gamma)$ are the connected components of the graph $\Gamma_{B}=\left(V(\Gamma), \gamma^{-1}(B)\right)$. For each $c \in \Delta_{n}$, we set $\hat{c}=\Delta_{n}-\{c\}$.

The vertices of $\Gamma$ whose degree is strictly less than $n+1$ are called the boundary vertices of $\Gamma$; if $\Gamma$ has no boundary vertices, i.e. if $\Gamma$ is regular of degree $n+1$, then $(\Gamma, \gamma)$ is said to be an $(n+1)$-coloured graph without boundary.

The graph' $(\Gamma, \gamma)$ is called regular with respect to the colour $c \in \Delta_{n}$ iff $\Gamma_{\hat{c}}$ is regular of degree $n$. From now on, we only consider $(n+1)$-coloured graphs which are regular with respect to the "last" colour $n$.

If $(\Gamma, \gamma)$ is an $(n+1)$-coloured graph, the boundary graph $\left(\partial \Gamma,{ }^{\partial} \gamma\right)$ is defined in the following way:

- the vertices of $\partial \Gamma$ are the boundary vertices of $\Gamma$;

- two vertices of $\partial \Gamma$ are joined by a $c$-coloured edge iff they belong to the same $\{n, c\}$-residue of $(\Gamma, \gamma)$.

Given an $(n+1)$-coloured graph $(\Gamma, \gamma)$, let us denote by $g(\Gamma)$ the number of components of $\Gamma$; by convention we set $g(\emptyset)=1$. A connected $(n+1)$-coloured graph $(\Gamma, \gamma)$ is called contracted iff $g\left(\Gamma_{\hat{n}}\right)=1$ and, for every $c \in \Delta_{n-1}, g\left(\Gamma_{\hat{c}}\right)=g(\partial \Gamma)$.

Let $K$ be an $n$-dimensional pseudocomplex [HW]. The disjoint star $\operatorname{std}(s, K)$ of a simplex $s$ in $K$ is the disjoint union of the $n$-simplexes containing $s$, with re-identification of the $(n-1)$-simplexes containing $s$ and of all their faces; the disjoint link of $s$ in $K$ is the subcomplex $\operatorname{lkd}(s, K)=\{\tau \in \operatorname{std}(s, K) / s \cap \tau=\emptyset\}$.

A vertex-coloration on $K$ is a map $\xi: V(K) \rightarrow \Delta_{n}$ which is injective on every simplex of $K$. If $K$ is homogeneous, the pair $(K, \xi)$ is called a coloured $n$-complex.

From now on, for sake of conciseness, we often drop edge - and vertex - colorations, writing $\Gamma$ and $K$ instead of $(\Gamma, \gamma)$ and $(K, \xi)$.

There exists a correspondence between $(n+1)$-coloured graphs and coloured $n$-complexes. In fact, given an $(n+1)$-coloured graph $\Gamma$, we can construct a coloured $n$-complex $K(\Gamma)$ in the following way:

- take an $n$-simplex $\sigma(v)$ for each $v \in V(\Gamma)$ and label its vertices by $\Delta_{n}$ 
- for each $c \in \Delta_{n}$ and each pair $v, w$ of $c$-adjacent vertices in $\Gamma$, identify the $(n-1)$-faces of $\sigma(v)$ and $\sigma(w)$ opposite to the vertices labelled $c$, so that equally labelled vertices coincide.

The above construction can be easily reversed in order to associate an $(n+1)$-coloured graph $\Gamma(K)$ to each coloured $n$ complex $K$.

Note that, by construction, each $\left(\Delta_{n}-\left\{c_{0}, \ldots, c_{h}\right\}\right)$-residue $\Xi$ of $\Gamma$ corresponds to a unique $h$-simplex $s$ of $K(\Gamma)$, whose vertices are labelled by $\left\{c_{0}, \ldots, c_{h}\right\}$ and viceversa; moreover $K(\Xi)=\operatorname{lkd}(s, K(\Gamma))$. It is easy to see that $\Gamma(K(\Gamma))=\Gamma$; conversely $K(\Gamma(K))=K$ iff the disjoint star of every simplex in $K$ is strongly connected $\left[G_{1}\right]$. In this case $K$ is said to be representable.

If $|K(\Gamma)|$ (the polyhedron determined by $K(\Gamma)$ ) is a manifold, then $\partial(|K(\Gamma)|)=|K(\partial \Gamma)|$ and we say that $|K(\Gamma)|$ is represented by $\Gamma$. Moreover $|K(\Gamma)|$ is orientable iff $\Gamma$ is bipartite.

A contracted $(n+1)$-coloured graph representing a manifold $M$ is called a crystallization of $M$.

For a general survey on manifold representation theory by means of edge-coloured graphs, see [FGG].

In $\left[G_{2}\right],\left[G_{4}\right]$ a particular concept of imbedding of a coloured graph into a surface is defined. This naturally leads to the definition of an invariant for manifolds, the regular genus, which extends to dimension $n$ the classical notion of genus of a surface.

In $\left[\mathrm{G}_{3}\right]$ the regular genus of a closed 3-manifold is proved to coincide with its classical Heegaard genus $([\mathrm{H}]$ and $[\mathrm{He}])$. In this paper we extend the result, for the orientable case, to manifolds with boundary. In particular, by means of branched coverings techniques, we prove that the Heegaard genus (see [M]) and the regular genus of an orientable 3-manifold with boundary coincide. About non-orientable 3-manifolds with boundary, we only know that the Heegaard genus is less than or equal to the regular genus, since part of the proof of the main result of this paper (Lemma 1 and 3) still holds. Unfortunately we lack, for the non-orientable case, results similar to those of $[\mathrm{M}]$, which are required in Lemma 2 to prove the opposite inequality. 


\section{REGULAR GENUS}

Let $\Gamma$ be an $(n+1)$-coloured graph (with boundary), regular with respect to the colour $n$.

We call extended graph associated to $\Gamma$ the $(n+1)$-coloured graph $\Gamma^{*}$ such that:

- $V\left(\Gamma^{*}\right)=V(\Gamma) \cup V^{*}$, where $V^{*}$ is in one-to-one correspondence with $V(\partial \Gamma)$

- $E\left(\Gamma^{*}\right)=E(\Gamma) \cup E^{*}$, where $E^{*}$ is the set of all possible $n$-coloured edges whose endpoints are a boundary-vertex of $\Gamma$ and its correspondent vertex in $V^{*}$.

A regular imbedding of $\Gamma$ into a surface (with boundary) $F$, is an imbedding $\iota^{*}$ of $\Gamma^{*}$ into $F$, satisfying the following conditions:

(a) $\iota^{*}\left(V^{*}\right)=\partial F \cap \iota^{*}\left(\left|\Gamma^{*}\right|\right)$

(b) the connected components of (int $F$ ) $-\iota^{*}\left(\left|\Gamma^{*}\right|\right.$ ) (the regions of the imbedding) are open balls;

(c) the boundary of any region $\mathcal{R}$ of $\iota^{*}$ is either the image of a cycle of $\Gamma^{*}$ (internal region) or the union of the image $\mathcal{R}^{\prime}$ of a path in $\Gamma^{*}$ and an arc $\mathcal{R}^{\prime \prime}$ of $\partial F$, the intersection consisting of two (possibly coincident) vertices of $\iota^{*}\left(V^{*}\right)$ (boundary region);

(d) there exists a cyclic permutation $\varepsilon=\left(\varepsilon_{0}, \varepsilon_{1}, \ldots, \varepsilon_{n}\right)$ of $\Delta_{n}$ such that for each internal region $\mathcal{R}$ (resp. boundary region $\mathcal{R}^{\prime} \cup \mathcal{R}^{\prime \prime}$ ), the edges of $\partial \mathcal{R}$ (resp. of $\mathcal{R}^{\prime}$ ) are alternatively coloured $\varepsilon_{i}$ and $\varepsilon_{i+1}$ $\left(i \in Z_{n+1}\right)$.

According to $\left[\mathrm{G}_{2}\right]$ and $\left[\mathrm{G}_{4}\right]$, for each cyclic permutation $\varepsilon=\left(\varepsilon_{0}, \varepsilon_{1}\right.$, $\ldots, n$ ) of $\Delta_{n}$, there exists a surface (with boundary) $F_{\varepsilon}$ and a regular imbedding $i_{\varepsilon}$ of $\Gamma$ into $F_{\varepsilon}$; moreover $F_{\varepsilon}$ is orientable iff $\Gamma$ is bipartite. $F_{\varepsilon}$ is called the regular surface associated to $\Gamma$ and $\varepsilon$. tion 4]):

If $F_{\varepsilon}$ is orientable, we have the following formula (see $\left[\mathrm{G}_{4}\right.$ Proposi- 


$$
\begin{aligned}
& \operatorname{genus}\left(F_{\varepsilon}\right)=1-\frac{1}{2}\left[\sum_{i \in Z_{n+1}} g_{\varepsilon_{i} \varepsilon_{i+1}}(\Gamma)+\right. \\
& \left.+\frac{1}{2}(1-n)(p(\Gamma)-\bar{p}(\Gamma))+(2-n) \frac{\bar{p}(\Gamma)}{2}\right]-\frac{1}{2} \partial g_{\varepsilon_{0} \varepsilon_{n-1}}(\Gamma)
\end{aligned}
$$

where $g_{i j}(\Gamma)$ (resp. ${ }^{\partial} g_{i j}(\Gamma)$ ) is the number of cycles of $\Gamma_{\{i, j\}}$ (resp. of $\left.\partial \Gamma_{\{i, j\}}\right)$ and $p(\Gamma)($ resp. $\bar{p}(\Gamma))$ is the order of $\Gamma$ (resp. of $\left.\partial \Gamma\right)$. Of course an analogous formula holds for non-orientable regular surfaces.

Set $\rho_{\varepsilon}(\Gamma)=\operatorname{genus}\left(F_{\varepsilon}\right)$. The regular genus $\rho(\Gamma)$ of $\Gamma$ is defined as the minimum $\rho_{\varepsilon}(\Gamma)$ among all cyclic permutations $\varepsilon$ of $\Delta_{n}$.

Given an $n$-manifold $M$ the regular genus of $M$ is the non-negative integer:

$$
\mathcal{G}(M)=\min \{\rho(\Gamma) / \Gamma \text { represents } M\}
$$

If $\Gamma$ is a 4 -coloured graph representing a 3 -manifold $M$, let us describe an effective construction of $F_{\boldsymbol{\varepsilon}}$.

Let $K_{\varepsilon}^{\prime}$ (resp. $\left.K_{\varepsilon}^{\prime \prime}\right)$ be the 1-dimensional subcomplex of $K$ generated by the vertices coloured $\left(\varepsilon_{1}, 3\right)$ (resp. $\left.\left(\varepsilon_{0}, \varepsilon_{2}\right)\right)$. Denote by $H_{\varepsilon}$ the largest 2-dimensional subcomplex of $S d K$ (where $S d$ means "first barycentric subdivision") disjoint from $S d K_{\varepsilon}^{\prime} \cup S d K_{\varepsilon}^{\prime \prime}$. Then $F_{\varepsilon}=\left|H_{\varepsilon}\right|$ is the regular surface associated to $\Gamma$ and $\varepsilon\left[\mathrm{G}_{4}\right]$. Moreover $F_{\varepsilon}$ is an orientable surface with at least one boundary component on each component of $\partial M$; if $\Gamma$ is a crystallization of $M$, then each boundary component of $F_{\varepsilon}$ lies on a different component of $\partial M$ (see Proposition 12 of $\left[\mathrm{G}_{4}\right]$ ).

Note that $H_{\varepsilon}$ splits $K(\Gamma)$ into two subcomplexes $N_{\varepsilon}^{\prime}$ and $N_{\varepsilon}^{\prime \prime}$ which admit $S d K_{\varepsilon}^{\prime}$ and $S d K_{\varepsilon}^{\prime \prime}$ as spines and such that $N_{\varepsilon}^{\prime} \cap N_{\varepsilon}^{\prime \prime}=\partial N_{\varepsilon}^{\prime} \cap \partial N_{\varepsilon}^{\prime \prime}=$ $H_{\varepsilon}$. Let us set $\mathcal{A}_{\varepsilon}^{\prime}=\left|N_{\varepsilon}^{\prime}\right|, \mathcal{A}_{\varepsilon}^{\prime \prime}=\left|N_{\varepsilon}^{\prime \prime}\right|$.

The pair $\left(\mathcal{A}_{\varepsilon}^{\prime}, \mathcal{A}_{\varepsilon}^{\prime \prime}\right)$ is called the regular splitting associated to $\Gamma$ and $\varepsilon$. 
Given a 3-manifold $M$ by [B Theorem 1] the minimum genus can always be obtained by a crystallization of $M$. In this case we can set;

$$
\mathcal{G}(M)=\min \{\rho(\Gamma) / \Gamma \text { is a crystallization of } M\} .
$$

Moreover, if $M$ is closed, then $\mathcal{G}(M)=\mathcal{H}(M)$, where $\mathcal{H}$ denotes the Heegaard genus (see $\left[G_{3}\right]$ ).

\section{HEEGAARD SPLITTINGS AND DIAGRAMS}

A singular 3-manifold is a 3-dimensional polyhedron $N=|H|, H$ being a simplicial complex, such that for each vertex $v$ of $H$, the link $l k(v, H)$ is a combinatorial closed connected surface.

Note that if $K$ is a pseudocomplex and $|K|=N$, then $N$ is a singular 3-manifold iff for each vertex $v$ of $K$, the disjoint link $l k d(v, K)$ is a combinatorial closed connected surface. In fact, the first barycentric subdivision $K^{\prime \prime}$ of $K$ is a simplicial complex and $l k\left(v, K^{\prime \prime}\right)$ is isomorphic to a subdivision of $l k d(v, K)$.

Note also that if $N$ is a singular 3 -manifold and $|H|=N, H$ being a simplicial complex (resp. $|K|=N, K$ being a pseudocomplex), then for each $h$-simplex $\sigma^{h}$ of $H$ (resp. of $K$ ), with $h \geq 1$, the link $l k\left(\sigma^{h}, H\right)$ (resp. the disjoint link $\left.l k d\left(\sigma^{h}, K\right)\right)$ is a combinatorial $(3-h-1)$-sphere.

Hence, a polyhedron $N$ is a singular 3-manifold iff each point $x$ of $H$ has a neighbourhood (PL) homeomorphic to a cone over a closed connected surface. If such a surface is not a sphere, then $x$ is called a singular point of $N$.

Given a 3 -manifold $M$, we obtain a singular 3 -manifold $N=\hat{M}$ by capping off each component of $\partial M$ by a cone over it. Conversely, given a singular 3-manifold $N$, we denote by $\dot{N}$ the 3-manifold obtained from $N$ by removing small open neighbourhoods of its singular points. If $K$ is any (pseudo or simplicial) complex triangulating $N$, then $\bar{N}$ can be simply obtained by deleting the open stars of the singular vertices in a suitable subdivision of $K$.

Note that, if we avoid 3-manifolds with spherical boundary components, the correspondence $N \rightarrow \bar{N}$ becomes bijective. 
Let now $G$ be the 1-dimensional subset of $\mathbf{S}^{3}$ pictured in Figure 1. By [M], given a 3-manifold $M$, there exist an integer $h \geq 1$ and a transitive pair of permutations $\sigma, \tau \in \Sigma_{h}$ such that $M \cong \check{N}(\sigma, \tau)$, where $N(\sigma, \tau)$ is the $h$-fold covering of $\mathbf{S}^{3}$ branched over $G$, whose monodromy sends the two generators of $\pi_{1}\left(\mathbf{S}^{3}-G\right)$ determined by the oriented meridians of Figure 1, to $\sigma$ and $\tau$.

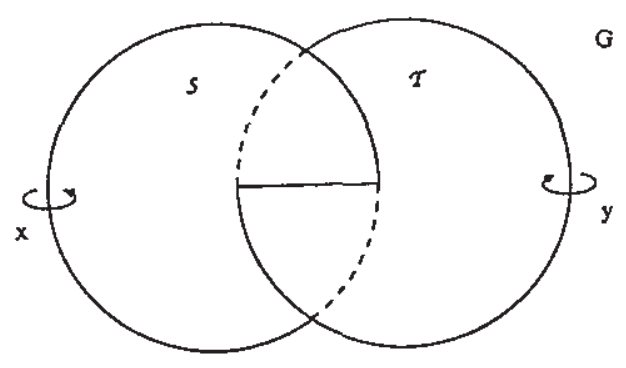

Figure 1

An effective construction of a triangulation $K(\sigma, \tau)$ of $N(\sigma, \tau)$ proceeds as follows:

- take $h$ copies $t_{1}, \ldots, t_{h}$ of the standard tetrahedron $t$ whose bidimensional faces are denoted by $S, \bar{S}$ and $T, \bar{T}$ as in Figure 2;

- for each $i=1, \ldots, h$ call $S_{i \sigma(i)}, \bar{S}_{i \sigma^{-1}(i)}, T_{i \tau(i)}, \bar{T}_{i \tau^{-1}(i)}$ the faces $S, \bar{S}, T, \tilde{T}$ (respectively) in the copy $t_{i}$;

- identify $S_{i j}$ with $\bar{S}_{j i}$ and $T_{i j}$ with $\bar{T}_{j i}$ by a linear homeomorphism respecting the edges $S \cap \bar{S}$ and $T \cap \bar{T}$.

Let $T_{g}$ be the closed orientable surface of genus $g$, where, $T_{0}$ stays for the 2-sphere $S^{2}$. By a proper handlebody of genus $g \geq 0$ we mean an (orientable) 3-manifold $X_{g}$, obtained by attaching $g 1$-handles on the boundary of the 3-dimensional disk $D^{3}$. Note that two such handlebodies are homeomorphic iff they have the same genus. The boundary $\partial X_{g}$ of $X_{g}$ is the closed orientable surface $T_{g}$. 


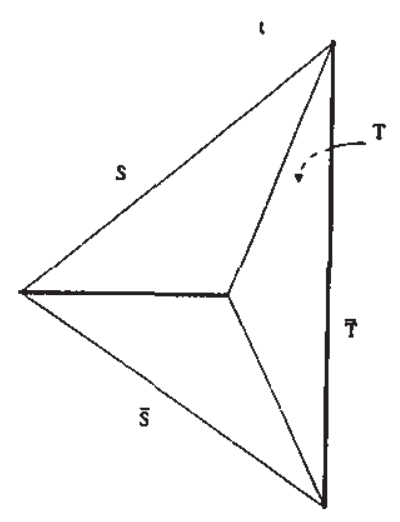

Figure 2.

By a hollow handlebody of genus $g \geq 0$ we mean an (orientable) 3manifold $X_{g}$ obtained from $T_{g} \times[0,1]$ by adding 2 - and 3 -handles along $T_{g} \times\{1\} ; T_{g} \times\{0\}$ is called the free boundary of $X_{g}$.

Remark 1. Note that a hollow handlebody $X_{g}$ of genus $g$ is proper iff its boundary $\partial X_{g}$ coincides with its free boundary $T_{g} \times\{0\}$.

In fact, if $X_{g}$ is proper, then $X_{g}=D^{3} \cup H_{1}^{(1)} \cup \ldots \cup H_{g}^{(1)}$, where for $1 \leq i \leq g, H_{i}^{(1)}$ is a 1 -handle on the boundary of $D^{3}$. By adding a collar on $\partial X_{g}=T_{g}$ and dualizing the handle presentation, we have $X_{g}=\left(T_{g} \times[0,1]\right) \cup H_{g}^{*} \cup \ldots \cup H_{1}^{*} \cup D^{3}$, where each $H_{i}^{*}$ is now a 2-handle on $T_{g} \times\{1\}$ and $D^{3}$ is a 3-handle.

Conversely, if $X_{g}$ is a hollow handlebody and $\partial X_{g}=T_{g} \times\{0\}$, then

$$
X_{g}=\left(T_{g} \times[0,1]\right) \cup H_{1}^{(2)} \cup \ldots \cup H_{r}^{(2)} \cup \tilde{H}_{1}^{(3)} \cup \ldots \cup \tilde{H}_{s}^{(3)}
$$


where, for $1 \leq i \leq r, H_{i}^{(2)}$ is a 2 -handle on $T_{g} \times\{1\}$ and for $1 \leq j \leq s$, $\tilde{H}_{j}^{(3)}$ is a 3 -handle. Hence, by dualizing the presentation and deleting a collar of the boundary, we obtain: $X_{g}=\tilde{H}_{1}^{*} \cup \ldots \cup \tilde{H}_{s}^{*} \cup H_{r}^{*} \cup \ldots \cup H_{1}^{*}$ where $\tilde{H}_{1}^{*}, \ldots, \tilde{H}_{s}^{*}$ are 0 -handles and $H_{r}^{*}, \ldots, H_{1}^{*}$ are 1 -handles. This proves that $X_{g}$ is proper and we can simplify the presentation in the following way: $X_{g}=D^{3} \cup \bar{H}_{1}^{(1)} \cup \ldots \cup \bar{H}_{r-s+1}^{(1)}$. Since $\partial X_{g}=T_{g}$, it follows that $r-s+1=g$.

A generalized Heegaard splitting of a 3-manifold $M$ is a pair $\left(X_{g}, Y_{g}\right)$ of hollow handlebodies of genus $g$ such that:

- $M=X_{g} \cup Y_{g}$

- $T_{g}=X_{g} \cap Y_{g}$ is the free boundary of both $X_{g}$ and $Y_{g}$.

The non-negative integer $g$ is called the genus of the splitting.

If at least one of the two hollow handlebodies is proper, then $\left(X_{g}\right.$, $Y_{g}$ ) is said to be a proper Heegaard splitting of $M$; in this case we always suppose that $X_{g}$ is proper.

The Heegaard genus of $M$ is defined to be the non-negative integer:

$$
\mathcal{H}(M)=\min \{g / M \text { admits a proper Heegaard splitting of genus } g\} \text {. }
$$

By Remark 1, the above definitions coincide with the homonymous ones given by Montesinos in $[\mathrm{M}]$; moreover, they generalize the classical ones given for closed manifolds.

A generalized Heegaard diagram is a triple $\left(T_{g} ; v ; w\right)$, where $v$ and $w$ are systems of simple closed curves on $T_{g}$.

Each generalized Heegaard splitting $\left(X_{g}, Y_{g}\right)$ of a 3 -manifold $M$ produces a generalized Heegaard diagram, whose systems of curves are the attaching spheres of the 2-handles on $X_{g}$ and $Y_{g}$.

Conversely, from a generalized Heegaard diagram $\left(T_{g} ; v, w\right)$ we can obtain a hollow handlebody $X$ (resp. $Y$ ) by considering $T_{g} \times[0,1]$ (resp. $T_{g} \times[-1,0]$ ) with 2 -handles attached on $T_{g} \times\{1\}$ (resp. $T_{g} \times\{-1\}$ ) according to $v$ (resp. to $w$ ) and possibly by capping off some of the spherical boundary components by 3-handles. If $M$ is the 3-manifold obtained from $X \cup Y$ by identifying their free boundaries, then $(X, Y)$ 
is a generalized Heegaard splitting of the 3 -manifold $M$. In this case we say that $\left(T_{g} ; v, w\right)$ represents $M$.

A generalized Heegaard diagram $\left(T_{g} ; v, w\right)$ is called a proper Heegaard diagram if its corresponding Heegaard splitting is proper.

Remark 2. If $\left(T_{g} ; v=\left(v_{1}, \ldots, v_{r}\right), w=\left(w_{1}, \ldots, w_{s}\right)\right)$ is a proper Heegaard diagram representing a 3-manifold $M$, then $r \geq g$; moreover, we can always find a subset $v^{\prime}$ of $v$, containing $g$ curves, such that $v^{\prime}$ is a complete system of meridian curves for $T_{g}$, i.e. $T_{g}-v^{\prime}$ is planar and connected. Since $\left(T_{g} ; v^{t}, w\right)$ still represents $M$, from now on we suppose $r=g$ (or, equivalently, $T_{g}-v$ to be planar connected).

Proposition 1. Every 3-manifold $M$ admits a proper Heegaard splitting.

Proof.' The first part of this proof adapts an analogous one in [S]. Let $K$ be a simplicial complex triangulating $M$ and $H_{2}$ a tubular neighbourhood of the dual 1-skeleton of $M$. Set $H_{1}=\overline{\overline{M-H_{2}}}$, then $H_{1}$ and $H_{2}$ are proper handlebodies whose intersection is a proper subset of their boundaries. More precisely, $\partial H_{1}$ and $\partial H_{2}$ are not identified along $\partial M$, but $\partial M \cap \partial H_{2}=\cup_{i} D_{i}$, where the $D_{i}^{\prime} s$ are disks.

Let $N_{i}=D^{2} \times[0,1]$ be a collar of $D_{i}$ in $H_{2}$ and $H_{1}^{\prime}$ the complex obtained by attaching the 2-handles $N_{i}$ along $\partial H_{1}$, respecting the identification 'between $\partial H_{1}$ and $\partial H_{2}$. Hence $H_{2}^{\prime}=\overline{H_{2}-\left(U_{i} N_{i}\right)}$ is a proper handlebody such that $H_{1}^{\prime} \cap H_{2}^{\prime}=\partial H_{1}^{\prime}=\partial H_{2}^{\prime}$ is a closed surface $S$. If $C$ is a collar of $S$ in $H_{2}^{\prime}$ then define $H_{1}^{\prime \prime}=H_{1}^{\prime} \cup C, H_{2}^{\prime \prime}=\overline{H_{2}^{\prime}-C}$.

$H_{2}^{\prime \prime}$ is a proper handlebody and $H_{1}^{\prime \prime}$ is a hollow handlebody obtained from $S \times[0,1]$ by attaching the 2-handles $N_{i}$ along $S \times\{0\}$.

If $M$ is a 3-manifold, then $M \cong \check{N}(\sigma, \tau)$ for a suitable transitive pair $(\sigma, \tau)$ of permutations. Let us describe a particular generalized Heegaard splitting of $M$ arising from $(\sigma, \tau)$.

Let us call $\mathcal{S}$ and $\mathcal{T}$ the two disks embedded in $S^{3}$ as in Figure 1. Let $F$ be the boundary of a tubular neighbourhood of $\partial S$ in $S^{3}$ and let $X$ and $Y$ be the closures of the two components of $S^{3}-F$. Then $X$ and $Y$ are regular neighbourhoods of $\partial \mathcal{S}$ and $\partial \mathcal{T}$ respectively and therefore $(X, Y)$ is a proper genus one Heegaard splitting of $S^{3}$. 
Let $\tilde{X}$ (resp. $\tilde{Y}$ ) be the hollow handlebody which is the preimage of $X$ (resp. of $Y$ ) by the branched covering map $N(\sigma, \tau) \rightarrow S^{3}$ and let $\tilde{F}$ be the preimage of $F$; then $(\tilde{X}, \tilde{Y})$ is a generalized Heegaard splitting (Theorem 10 of $[\mathrm{M}]$ ), which is called canonical Heegaard splitting of $\check{N}(\sigma, \tau)$.

If the canonical splitting is proper, one of the hollow handlebodies, $\tilde{X}$ say, is proper; hence, all singular points of $N(\sigma, \tau)$ lie in $\tilde{Y}$. It is easy to see that, in this case, the singular vertices in $K(\sigma, \tau)$ are the endpoints of some of the edges $T \cap \bar{T}$.

Let $H$ be a subgroup of $\Sigma_{h}(h \geq 1)$, generated by a certain set of permutations $\left\{\sigma_{1}, \ldots, \sigma_{\tau}\right\}$. We denote by $\left|\sigma_{1}, \ldots, \sigma_{r}\right|$ the number of orbits of the action of $H$ on $\{1,2, \ldots, h\}$.

By [M], the canonical Heegaard splitting of $\dot{N}(\sigma, \tau)$ is proper, i.e. $\ddot{X}$ (resp. $\tilde{Y}$ ) is a proper handlebody iff $\left|\sigma, \tau \sigma \tau^{-1}\right|=1$ (resp. $\left|\tau, \sigma \tau \sigma^{-1}\right|=$ 1).

A further result of $[\mathrm{M}]$ will be required later:

Proposition 2. Let $\left(T_{g} ; v, w\right)$ be a proper Heegaard diagram of a 3-manifold $M$, with $g>0$ and $w \neq \emptyset$. There is an algorithm which determines an integer $h \geq 1$ and two permutations $\sigma, \tau \in \Sigma_{h}$ such that

(i) $\check{N}(\sigma, \tau) \cong M$;

(ii) $\left|\sigma, \tau \sigma \tau^{-1}\right|=1$ (i.e. the canonical Heegaard splitting of $\check{N}(\sigma, \tau)$ is proper);

(iv) $|\sigma|=g=1+\frac{1}{2}(h-|[\sigma, \tau]|)$.

Remark 3. If $g=0$, then $M \cong S^{3}$ and we have directly $h=1$ and $\sigma=\tau=\mathrm{id}_{\{1\}}$. If $w=\emptyset$, then $M$ is a proper handlebody of genus $g$ and it is very easy to construct a new proper Heegaard diagram representing $M$ of genus $g+1$ and such that $w \neq \emptyset$.

\section{THE MAIN RESULT}

Proposition 3. For every 3-manifold $M$, we have $\mathcal{G}(M)=\mathcal{H}(M)$.

The proof requires three lemmas: 
Lemma 1. Let $\Gamma$ be a crystallization of a 3-manifold $M$. For each cyclic permutation $\varepsilon$ of $\Delta_{3}$, there exists a proper Heegaard splitting of $M$ whose genus is $\rho_{\varepsilon}(\Gamma)$.

Proof. Let $\left(\mathcal{A}_{\varepsilon}^{\prime}, \mathcal{A}_{\varepsilon}^{\prime \prime}\right)$ and $F_{\varepsilon}$ be the regular splitting and the regular surface associated to $\Gamma$ and $\varepsilon$. Note that $\mathcal{A}_{\varepsilon}^{\prime} \cap \partial_{i} M$ (where $\partial_{i} M$ is the $i$-th boundary component of $M$ ) is a single disk $B_{i}$ (since $\Gamma$ is a crystallization) such that $\partial B_{i}=\partial_{i} F_{\varepsilon} ;$ moreover $\mathcal{A}_{\varepsilon}^{\prime \prime} \cap \partial_{i} M=\partial_{i} M-\operatorname{int} B_{i}$.

Let us consider the closed surface $S_{\varepsilon}=F_{\varepsilon} \cup\left(\cup_{i} B_{i}\right)$ and a collar $C$ of $S_{\varepsilon}$ in $\mathcal{A}_{\varepsilon}^{\prime}$; define $Y=\mathcal{A}_{\varepsilon}^{\prime \prime} \cup C$ and $X=\overline{\mathcal{A}}_{\varepsilon}^{\prime}-C$. $X$ is a proper handlebody with $\partial X=C_{1}$ (where $C_{1}$ is the subset of $\partial C$ corresponding to $\left.S_{\varepsilon} \times\{1\}\right)$ and $X \cap Y=C_{1}$.

$Y$ is a hollow handlebody with free boundary $C_{1}$. In fact consider, for each edge $e_{i}$ of $K(\Gamma)$ whose endpoints are coloured by $\varepsilon_{1}$ and 3 , the 2-handle $H_{i}^{(2)}$ which is a regular neighbourhood of the dual 2-cell of $e_{i}$ (see Figure 3); $Y$ is obtained from $S_{\varepsilon} \times[0,1]$ by adding the $H_{i}^{(2)} s$ along $S_{\varepsilon} \times\{0\}$.

To complete the proof observe that the resulting proper Heegaard splitting $(X, Y)$ of $M$ has genus:

genus $\left(C_{1}\right)=$ genus $\left(S_{\varepsilon}\right)=$ genus $\left(F_{\varepsilon}\right)=\rho_{\varepsilon}(\Gamma)$.

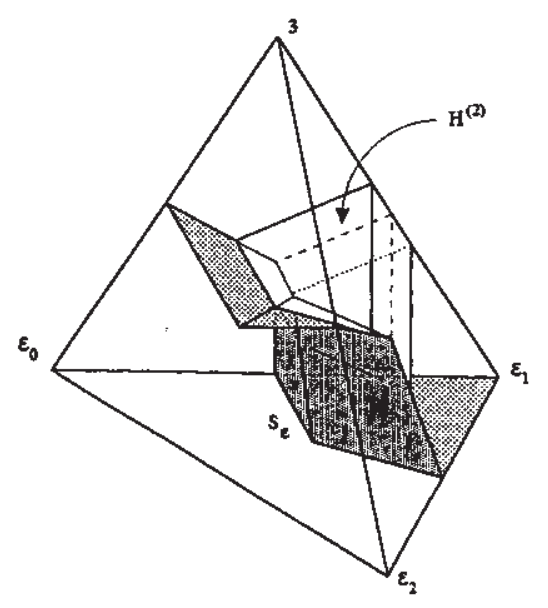

Figure 3. 
Lemma 2. Let $M$ be a 3-manifold which is not a proper handlebody and let $N=\hat{M}$ be the singular 3-manifold associated to $M$. There exists a 4-coloured graph without boundary $\Gamma$ representing $N$ such that:

- if $\varepsilon=(0,1,2,3)$, then $\rho_{\varepsilon}(\Gamma)=\mathcal{H}(M)$;

- all singular vertices of $K(\Gamma)$ are 0 -coloured.

Proof. Suppose that $\left(T_{g} ; v, w\right)$ is a proper Heegaard diagram representing $M$ such that $g=\mathcal{H}(M)$.

By Proposition 2, we can algorithmically determine $h \geq 1$ and $\sigma, \tau \in$ $\Sigma_{h}$ such that $\bar{N}(\sigma, \tau) \cong M,\left|\sigma, \tau \sigma \tau^{-1}\right|=1,|\sigma|=g=1+\frac{1}{2}(h-|[\sigma, \tau]|)$.

Consider the triangulation $K(\sigma, \tau)$ of $N(\sigma, \tau)$ described in section 3 and subdivide it in the following way (see $[\mathrm{Gr}]$ ):

- for each tetrahedron $t$, let $V_{S}$ (resp. $V_{T}$ ) be the barycenter of $S \cap \bar{S}$ (resp. $T \cap \bar{T}$ ), join $V_{S}$ and $V_{T}$ by an edge lying in the interior of $t$ and join $V_{S}$ (resp. $V_{T}$ ) with the endpoints of $T \cap \bar{T}$ (resp. $S \cap \bar{S}$ ).

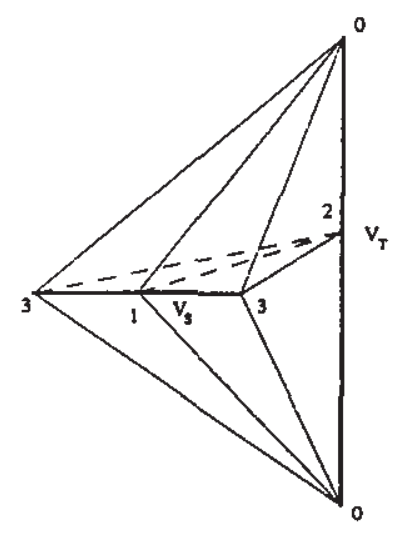

Figure 4.

Label now $V_{S}$ (resp. $V_{T}$ ) by colour 1 (resp. by 2 ) and the endpoints of $S \cap \bar{S}$ (resp. of $T \cap \bar{T}$ ) by 3 (resp. by 0 ) (see Figure 4), thus obtaining 
a representable pseudocomplex $K^{\prime}$. Let $\Gamma$ be its associated 4-coloured graph (without boundary).

Note that, by Proposition 2, the canonical Heegaard splitting of $\check{N}(\sigma, \tau)$ is proper; therefore all the singular vertices of $K^{\prime}$ are 0 -coloured.

Moreover $\sharp V(\Gamma)=4 h, g_{01}(\Gamma)=h, g_{12}(\Gamma)=|[\sigma, \tau]|=h+2-$ $2 g, g_{23}(\Gamma)=h, g_{03}(\Gamma)=h$.

If $\varepsilon=(0,1,2,3)$, formula ( $\left.{ }^{*}\right)$, for $n=3$, gives:

$$
\rho_{\varepsilon}(\Gamma)=1-\frac{1}{2}\left(g_{01}(\Gamma)+g_{12}(\Gamma)+g_{23}(\Gamma)-4 h\right)=g
$$

Let us recall some definitions and results about subdivisions of coloured graphs (see $\left[G_{5}\right]$ ).

Given a 4-coloured graph without boundary $\Gamma$, two colours $\alpha, \beta \in$ $\Delta_{3}$ and an $\alpha$-coloured vertex $w$ of $K(\Gamma)$, the bisection of $\Gamma$ of type $(\alpha, \beta)$ around $w$ is the 4-coloured graph $b \Gamma$ associated to the coloured complex $b K(\Gamma)$ obtained from $K(\Gamma)$ in the following way:

- consider the set $K_{\beta}(\Gamma)$ of all edges of $K(\Gamma)$ whose endpoints are $w$ and a $\beta$-coloured vertex and perform a stellar subdivision on each edge of $K_{\beta}(\Gamma)$;

- colour $w$ by $\beta$ and the barycenters of the elements of $K_{\beta}(\Gamma)$ by $\alpha$.

The coloration of $b K(\Gamma)$ agrees with that of $K(\Gamma)$ on the remaining vertices.

Let $e$ be an edge of $K(\Gamma)$ whose endpoints, $w_{\alpha}$ and $w_{\beta}$, are $\alpha$ - and $\beta$-coloured respectively; the trisection of $\Gamma$ of type $(\alpha, \beta)$ on $e$ is the 4coloured graph associated to the coloured complex obtained from $K(\Gamma)$ in the following way:

- perform two successive stellar subdivisions of $K(\Gamma)$ : the first on $e$, introducing a new vertex $w_{\alpha}^{\prime}$, the second on the edge of endpoints $w_{\alpha}^{\prime}$ and $w_{\alpha}$, introducing another vertex $w_{\beta}^{\prime}$;

- for $c \in\{\alpha, \beta\}$ colour $w_{c}^{\prime}$ by $c$, keeping the coloration of $K(\Gamma)$ for the remaining vertices. 
We shall call trisection of type $(\alpha, \beta)$ around the $\alpha$-coloured vertex $w$ the graph $t \Gamma$ associated to the complex $t K(\Gamma)$, obtained by performing trisections of type $(\alpha, \beta)$ on all edges of $K(\Gamma)$, having $w$ as endpoint.

If $\varepsilon$ is a cyclic permutation of $\Delta_{3}$, we have (see Proposition 7.1 and 7.2 of $\left.\left[\mathrm{G}_{5}\right]\right)$ :

- if $\alpha$ and $\beta$ are not consecutive in $\varepsilon$ then $\rho_{\varepsilon}(t \Gamma)=\rho_{\varepsilon}(\Gamma)=\rho_{\varepsilon}(b \Gamma)$;

- if $\alpha$ and $\beta$ are consecutive in $\varepsilon$ then $\rho_{\varepsilon}(b \Gamma)=\rho_{\varepsilon}(\Gamma)+g\left(\Lambda_{w}\right)+$ $g_{\beta \alpha^{\prime}}\left(\Lambda_{w}\right)-1$, where $\Lambda_{w}$ is the $\hat{\alpha}$-residue of $\Gamma$ representing $l k d(w$, $K(\Gamma))$ and $\alpha^{\prime}$ is the colour non-consecutive to $\alpha$ in $\varepsilon$.

Lemma 3. Let $\Gamma$ be a 4-coloured graph without boundary representing a singular 3-manifold $N$ such that all singular vertices in $K(\Gamma)$ are 0 -coloured. If $\varepsilon=(0,1,2,3)$, there exists a \&-coloured graph with boundary $\bar{\Gamma}$, regular with respect to 3 , representing $\bar{N}$ and such that $\rho_{\varepsilon}(\bar{\Gamma})=\rho_{\varepsilon}(\Gamma)$.

Proof. If $w$ is a ( 0 -coloured) singular vertex of $K(\Gamma)$, let $\Gamma^{(1)}$ be the trisection of $\Gamma$ of type $(0,2)$ around $w$. Hence $\rho_{\varepsilon}\left(\Gamma^{(1)}\right)=\rho_{\varepsilon}(\Gamma)$ because 0 and 2 are not consecutive in $\varepsilon$.

Consider now the bisection $\Gamma^{(2)}$ of the previous graph of type $(0,3)$ around $w$.

The genus of $\Gamma^{(2)}$ changes according to the following formula:

$\rho_{\varepsilon}\left(\Gamma^{(2)}\right)=\rho_{\varepsilon}\left(\Gamma^{(1)}\right)+\rho\left(\Lambda_{w}\right)+g_{23}\left(\Lambda_{w}\right)-1=\rho_{\varepsilon}(\Gamma)+\rho\left(\Lambda_{w}\right)+g_{23}\left(\Lambda_{w}\right)-1$

where $\Lambda_{w}$ is the $\hat{0}$-residue of $\Gamma^{(1)}$ representing $l k d\left(w, K\left(\Gamma^{(1)}\right)\right)$. Note that $w$ is now 3-coloured. Perform finally a trisection of type $(3,1)$ around $w$, obtaining a 4-coloured graph $\Gamma^{(3)}$, with $\rho_{\varepsilon}\left(\Gamma^{(3)}\right)=\rho_{\varepsilon}\left(\Gamma^{(2)}\right)$.

Delete now from $\Gamma^{(3)}$ the $\hat{3}$-residue $\Xi$ representing $l k d\left(w, K\left(\Gamma^{(3)}\right)\right)$ and the "hanging" 3 -coloured edges and call $\Gamma^{r}$ the resulting 4-coloured graph with boundary. Clearly $\Gamma^{\prime}$ is regular with respect to the colour 3 .

Note that:

(1) $g_{i j}\left(\Gamma^{\prime}\right)=g_{i j}\left(\Gamma^{(3)}\right)-g_{i j}(\Xi) \quad \forall i, j \in\{0,1,2\}$ 
(2) $g_{3 i}\left(\Gamma^{\prime}\right)=g_{3 i}\left(\Gamma^{(3)}\right)-\frac{1}{2} \bar{p}\left(\Gamma^{\prime}\right) \quad \forall i \in\{0,1,2\}$

(3) $p\left(\Gamma^{(3)}\right)=p\left(\Gamma^{\prime}\right)+\bar{p}\left(\Gamma^{\prime}\right)$

(4) $\bar{p}\left(\Gamma^{\prime}\right)=p(\Xi)$

(5) ${ }^{a} g_{02}\left(\Gamma^{\prime}\right)=g_{02}(\Xi)$.

By formula $\left(^{*}\right)$ applied to $\Gamma^{\prime}$ we have:

$$
\begin{gathered}
\rho_{\varepsilon}\left(\Gamma^{\prime}\right)=1-\frac{1}{2}\left[g_{01}\left(\Gamma^{\prime}\right)+g_{12}\left(\Gamma^{\prime}\right)+g_{23}\left(\Gamma^{\prime}\right)+g_{03}\left(\Gamma^{\prime}\right)-\left(p\left(\Gamma^{\prime}\right)-\bar{p}\left(\Gamma^{\prime}\right)\right)-\right. \\
\left.-\frac{1}{2} \bar{p}\left(\Gamma^{\prime}\right)\right]-\frac{1}{2} \partial g_{02}\left(\Gamma^{\prime}\right)
\end{gathered}
$$

By applying formula $(*)$, for $n=2$, to the 3-coloured graph $\Xi$ and the permutation $\varepsilon^{\prime}=(0,1,2)$, we obtain:

$$
\rho_{\varepsilon^{\prime}}(\Xi)=1-\frac{1}{2}\left[g_{01}(\Xi)+g_{12}(\Xi)+g_{02}(\Xi)-\frac{1}{2} p(\Xi)\right]
$$

By adding $\left({ }^{* *}\right)$ and $\left({ }^{* * *}\right)$ and making use of $(1),(2),(3)$ we have:

$\rho_{\varepsilon}\left(\Gamma^{\prime}\right)+\rho_{\varepsilon^{\prime}}(\Xi)=\rho_{\varepsilon}\left(\Gamma^{(3)}\right)+1-\frac{1}{2}\left[\frac{1}{2} \bar{p}\left(\Gamma^{\prime}\right)+g_{02}(\Xi)-\frac{1}{2} p(\Xi)\right]-\frac{1}{2} \partial g_{02}\left(\Gamma^{\prime}\right)$

By substituting equalities (4) and (5) we obtain:

$$
\begin{aligned}
\rho_{\varepsilon}\left(\Gamma^{\prime}\right)+\rho_{\varepsilon^{\prime}}(\Xi) & =\rho_{\varepsilon}\left(\Gamma^{(3)}\right)+1-\frac{1}{2}\left[\frac{1}{2} \bar{p}\left(\Gamma^{\prime}\right)+{ }^{\partial} g_{02}\left(\Gamma^{\prime}\right)-\frac{1}{2} \bar{p}\left(\Gamma^{\prime}\right)\right]- \\
& -\frac{1}{2}{ }^{\partial} g_{02}\left(\Gamma^{\prime}\right)=\rho_{\varepsilon}\left(\Gamma^{(3)}\right)+1-{ }^{\partial} g_{02}\left(\Gamma^{\prime}\right) .
\end{aligned}
$$

Finally formula (') gives:

$$
\rho_{\varepsilon}\left(\Gamma^{\prime}\right)=\rho_{\varepsilon}(\Gamma)+\rho\left(\Lambda_{w}\right)+g_{23}\left(\Lambda_{w}\right)-\rho_{\varepsilon^{\prime}}(\Xi)-{ }^{\partial} g_{02}\left(\Gamma^{\prime}\right)
$$


Note that both $g_{23}\left(\Lambda_{w}\right)$ and ${ }^{8} g_{02}\left(\Gamma^{\prime}\right)$ equal the number of edges in $K(\Gamma)$ whose endpoints are $w$ and a 1 -coloured vertex. Moreover, since $\Xi$ and $\Lambda_{w}$ are 3-coloured graphs, they admit a unique regular imbedding, namely the one in the surface $|l k d(w, K(\Gamma))|$, which both represent (see $\left[G_{4}\right]$ Corollary 5]), i.e. $\rho\left(\Lambda_{w}\right)=\rho(\Xi)=\rho_{\varepsilon^{\prime}}(\Xi)$. Hence $\rho_{\varepsilon}\left(\Gamma^{\prime}\right)=\rho_{c}(\Gamma)$.

By repeating the above procedure for all the singular vertices of $K(\Gamma)$, we obtain the required 4-coloured graph $\bar{\Gamma}$.

Proof of Proposition 3. If $\Gamma$ is a crystallization of $M$, then, by Lemma 1, we have $\mathcal{H}(M) \leq \rho_{\varepsilon}(\Gamma)$, for every choice of $\varepsilon$ and, by [B Theorem 1], it follows $\mathcal{H}(M) \leq \mathcal{G}(M)$.

If $M$ is a proper handlebody of genus $g$, then $\mathcal{G}(M)=g$ (see [ $\mathrm{G}_{4} \mathrm{pg}$. 276]). Since $\operatorname{rank}(M) \leq \mathcal{H}(M)$, we have $g \leq \mathcal{H}(M)$. Hence $g=\mathcal{H}(M)$.

Suppose now that $M$ is not a proper handlebody and let $N=\hat{M}$ be its associated singular manifold. Then the 4-coloured graph $\Gamma$ obtained by Lemma 2 satisfies the condition of Lemma 3. By applying Lemma 3 to $\Gamma$, we obtain a 4-coloured graph $\bar{\Gamma}$ representing $M$ such that $\rho_{\varepsilon}(\bar{\Gamma})=$ $\rho_{\varepsilon}(\Gamma)=\mathcal{H}(M)$. Hence $\mathcal{G}(M) \leq \rho_{\varepsilon}=\mathcal{H}(M)$.

Remark 4. Let $N$ be a singular 3-manifold and let $G^{0}(N)$ denote the set of all 4-coloured graphs $\Gamma$, representing $N$, such that the singular vertices of $K(\Gamma)$ are 0-coloured. Note that, by Lemma $2, G^{0}(N)$ is nonempty. The regular genus of $N$ is, by definition, the non-negative integer:

$$
\mathcal{G}(N)=\min \left\{\rho(\Gamma) / \Gamma \in G^{0}(N)\right\} .
$$

By Lemma 3 and Proposition 3, we have $\mathcal{G}(N) \geq \mathcal{H}(\check{N})=\mathcal{G}(\breve{N})$. If $\check{N}$ is not a proper handlebody, Lemma 2 gives $\mathcal{G}(N) \leq \mathcal{H}(\check{N})$. If $\dot{N}$ is a proper handlebody of genus $g$, the same inequality can be obtained by directly constructing a 4-coloured graph, of genus $g$, representing $N$ (see [FG]). Hence $\mathcal{G}(N)=\mathcal{H}(\check{N})=\mathcal{G}(\check{N})$ for every singular 3-manifold $N$. 


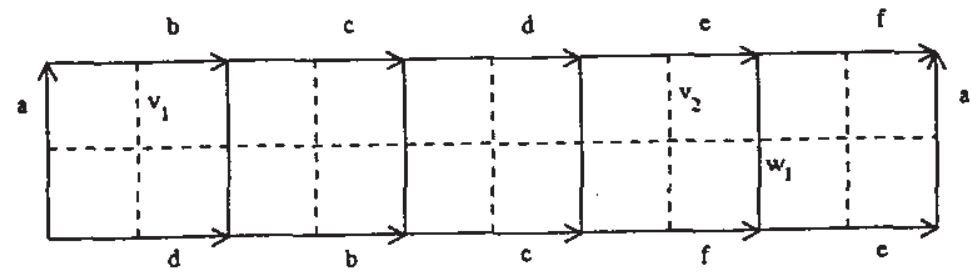

Figure 5 .
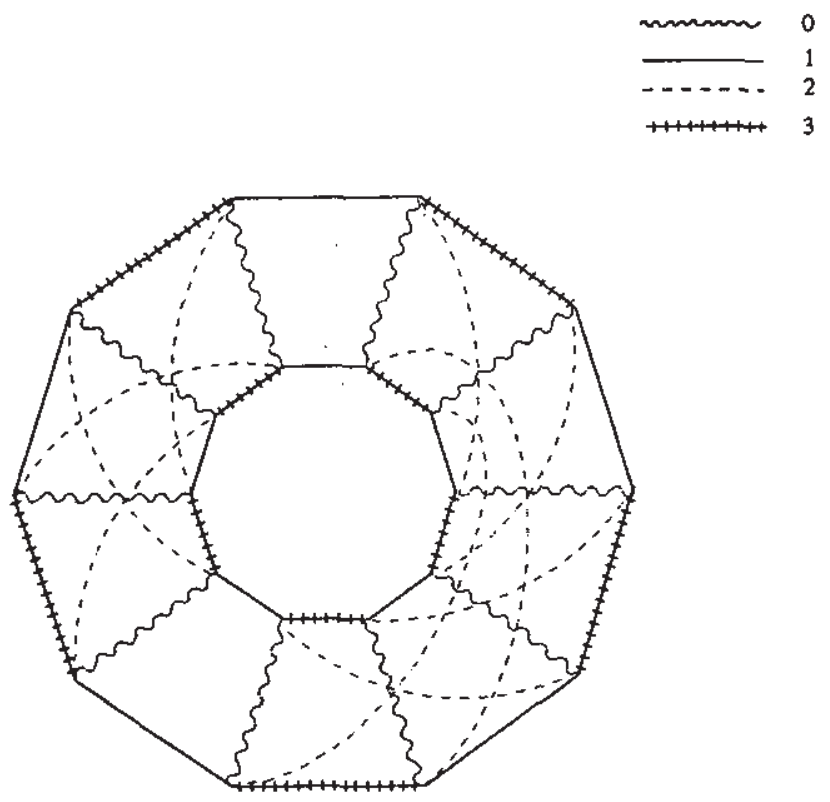

Figure 6 .

An example. The genus of the exterior of the trefoil knot. Let $M$ be the exterior of the trefoil knot. In Figure 5 a proper Heegaard diagram 
for $M$ is shown (see example 2 of [M]). By applying the algorithm of Proposition 2, we have: $\sigma=(123)(45)$ and $\tau=(12345)$. Figure 6 shows the 4-coloured graph $\Gamma$ obtained by using Lemma 2 . Its genus is $\rho(\Gamma)=\rho_{\varepsilon}(\Gamma)=2$, with $\varepsilon=(0,1,2,3)$. Therefore $\mathcal{G}(M)=\mathcal{H}(M) \leq 2$.

Actually $\mathcal{G}(M)=\mathcal{H}(M)=2$, since any genus one 3-manifold whose boundary is a torus, is homeomorphic to a solid torus (see also the final remark of $[\mathrm{C}])$. Moreover, the given Heegaard diagram describes the only genus two proper Heegaard splitting representing $M$ (see [BRZ]).

\section{References}

[B] Bandieri, P., A note on the genus of 3-manifolds with boundary, Ann. Univ. Ferrara - Sez. VII - Sc. Mat. vol. XXXV (1989), 163-175.

[BRZ] Boileau, M., Rost, M. and Zieschang, H., Décompositions de Heegaard des éxtérieurs des noeuds toriques et des variétés de Seifert associées, C.R. Acad. Sci. Paris 302, Sèrie I, 18 (1986), 661-664.

[C] Casali, M.R., A combinatorial characterization of 4-dimensional handlebodies, Forum Math. 4 (1992), 123-134.

[FG] Ferri, M. and Gagliardi, C., A characterization of punctured nspheres, Yokohama Math. Journal 33 (1985), 29-38.

[FGG] Ferri, M., Gagliardi, C. and Grasselli, L., A graph-theoretical representation of $P L$-manifolds - A survey on crystallizations, Aeq. Math. 31 (1986), 121-141.

[G $\left.\mathrm{G}_{1}\right]$ Gagliardi, C., A combinatorial characterization of 3-manifolds crystallisations, Boll. Un. Mat. Ital. 16-A (1979), 441-449.

$\left[\mathrm{G}_{2}\right]$ Gagliardi, C., Regular imbeddings of edge-coloured graphs, Geom. Dedicata 11 (1981), 397-414.

$\left[\mathrm{G}_{3}\right]$ Gagliardi, C., Extending the concept of genus to dimension n. Proc. Amer. Math. Soc. 81 (1981), 473-481.

[G $\left.{ }_{4}\right]$ Gagliardi, C., Regular genus: the boundary case, Geometriae Dedicata 22 (1987), 261-281.

[G $\left.\mathrm{G}_{5}\right]$ Gagliardi, C., On a class of 3-dimensional polyhedra, Ann. Univ. Ferrara, Sez. VII, Sc. Mat., vol. XXXIII (1987), 51-88. 
[Gr] Grasselli, L., 3-Manifolds Spines and Bijoins, Revista Matematica de la Universidad Complutense de Madrid vol. 3 n. 2-3 (1990), 165-179.

[H] Heegaard, P., Forstudier til topologisk teori för de algebraiske Sammenhäeng, Nordiske Forlag Ernst Bojesen, Copenhagen (1989); french translation: Bull Soc. Math. France 44 (1916), 161-212.

[He] Hempel, J., 3-Manifolds, Princeton University Press (1976).

[HW] Hilton, P.J. and Wylie, S., An introduction to Algebraic Topology - Homology Theory, Cambridge University Press (1960).

[M] Montesinos, J.M., Representing 3-manifolds by a universal branching set, Math. Proc. Cam. Phil. Soc. 94 (1983), 109-123.

[S] Stillwell, J., Classical Topology and Combinatorial Group Theory, Springer-Verlag (1980).

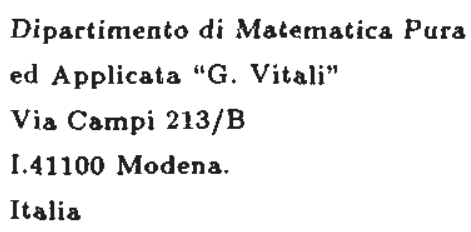

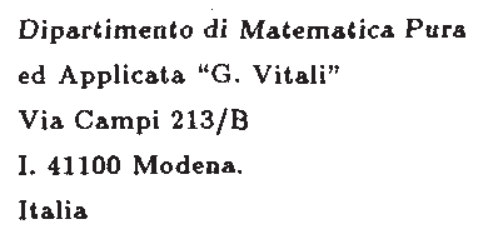

